\title{
Theo Blick - ein Mann der ersten Stunde
}

Keine Angst - Theo lebt! Das ist kein Nachruf, sondern eine kleine Laudatio für ihn, die er noch zu Lebzeiten entgegennehmen soll! Anlass ist sein Ausscheiden aus dem Vorstand der Arachnologischen Gesellschaft (AraGes) dem er 8 Jahre, d.h. seit der Gründung der Gesellschaft angehörte.

Will man Theos Rolle und Arbeit wirklich würdigen, dann sollte man wohl die Jahre vor der Gründung der AraGes näher betrachten. Theo Blick stieß zu einer Zeit zu den deutschen Arachnologen, in welcher ein bis dahin eher "elitärer" (man verzeihe mir diese Vereinfachung!!), überwiegend von Universitäts- und Museumswissenschaftlern dominierter Kreis (Taxonomen, Evolutionsforscher, Physiologen, Ökologen) sich schier "explosionsartig" um junge Leute erweiterte, die mehr anwendungsbezogen für Behörden und Naturschutzorganisationen faunistische Erhebungen der Spinnenfauna durchführten. Diese Untersuchungen wurden einerseits in der "ernsthaften Wissenschaft" nicht übermäßig ernst genommen (hier muss man die "ehemalige DDR" deutlich ausnehmen - die Faunistik hatte dort einen hohen, ihrer Wichtigkeit entsprechenden Stellenwert!) und fanden dementsprechend auch nur schwer ein Veröffentlichungsforum, andererseits litten die Bearbeiter unter dem Mangel an veröffentlichten Daten - sie sollten doch bewerten(!): Häufigkeit, Verbreitung, Bestandsentwicklung, Ökologie etc. Die Forderung, Schubladen, Archive und Aktenschränke von Bearbeitern, Instituten und Behörden zu öffnen, die Daten aller Bearbeiter zugänglich zu machen, zitierfähig zu veröffentlichen, zusammenzuführen, wurde erhoben, wo immer faunistisch arbeitende Arachnologen beieinander saßen, lag sozusagen allenthalben in der Luft. Hier musste einer wie Theo Blick kommen, jemand, der sich mit höchstem persönlichen Einsatz für ein Anliegen engagiert und der sein Umfeld mitreißen kann: Für Theo gab es (gibt es?) nie ein: „Man sollte/müsste mal diskutieren/überlegen/andenken, ob und wie....". Theo unterbreitete immer fertig formulierte Vorschläge und Strategien und bat nur noch um Stellungnahmen, Anregungen, Verbesserungsvorschläge. Natürlich hat er damit alle Kollegen, Mitarbeiter, Mitstreiter permanent

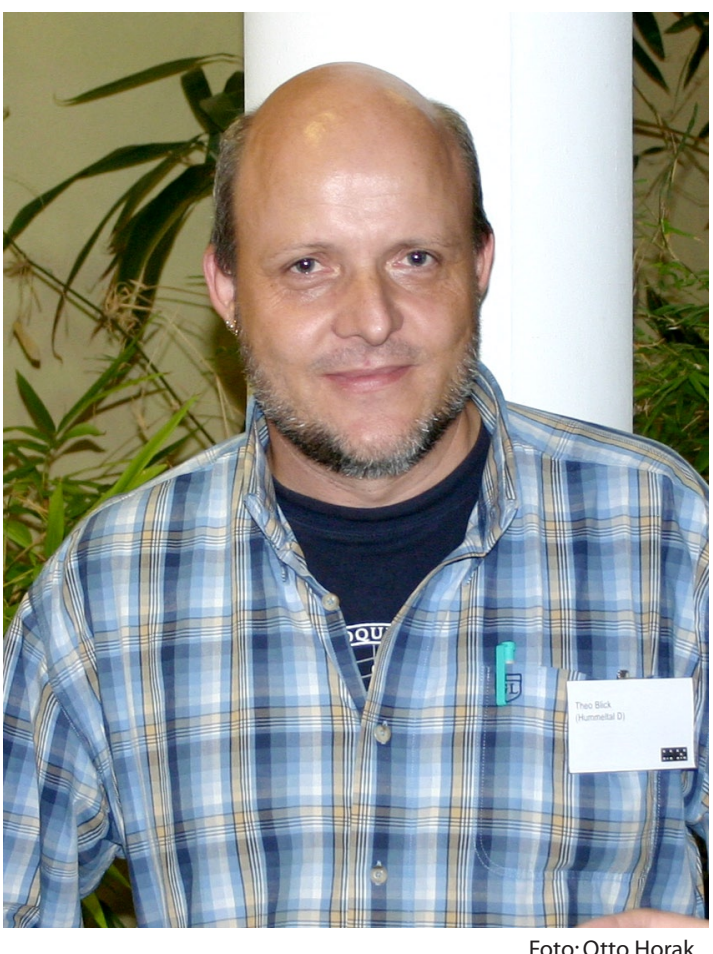

unter Duck gesetzt und zur Arbeit "geprügelt"! Der Erfolg allerdings hat ihn bestätigt - innerhalb eines Jahres war enorm viel passiert:

Während Theo noch in einem (privaten) Brief anfangs 1989 seine "Bereitschaft" bekundete, sich als Verhandlungspartner mit dem LfU (Landesamt für Umweltschutz, Bayern) zur Verfügung zu stellen, lag Ende 1989 bereits ein offizieller Auftrag vom LfU für die (honorierte) Erstellung einer Bayern-Checklist vor. Vorbereitet durch zwei lange(!) Rundbriefe ab August 89 (mit Adressenliste aller in Bayern arbeitenden Arachnologen, mit fertiger 6-seitiger Literaturliste für Bayern(!) usw.) fand am 2.12.1989 ein Treffen der bayerischen Arachnologen (18 Teilnehmer, 2 davon aus dem "befreundeten Ausland" Baden-Württemberg und Hessen) statt, in dessen Verlauf die "Arachnologische Arbeitsgemeinschaft Bayern" (AAB) gegründet wurde (wenn man so will, die "Keimzelle" der SARA) - der erste "institutionalisierte Spinnenverein" mit Jahresbeitrag, Rundbriefen, (geplanten) jährlichen Treffen, mit offiziellem Sprecher und Kontakt- 
mann zur Öffentlichkeit (Theo Blick) und - ganz wichtig - mit "Mitteilungen der AAB", einem internen Veröffentlichungsforum faunistischer Daten zum vertraulichen Gebrauch. Das 2. und 3. Treffen der AAB folgten Schlag auf Schlag im Juni und Oktober 1990, im März 90 wurde parallel die südwestdeutsche Arbeitsgemeinschaft in Stuttgart gegründet. Welch großes Bedürfnis nach Kommunikation und Austausch bestand, zeigte das rege und überregionale Interesse an diesen "lokalen" Treffen (Schweiz, Österreich, Hessen, Berlin, Sachsen). Die Zusammenlegung von bayerischer und südwestdeutscher Arge zur Süddeutschen Arachnologischen Arbeitsgemeinschaft SARA am 24.11.1990 in Stuttgart war deshalb nur folgerichtig. Und ein vordringlicher Punkt auf der Wunschliste der Faunisten wurde bei diesem Treffen gleich mit "abgearbeitet": die Gründung einer Zeitschrift, der "Arachnologischen Mitteilungen", die sich innerhalb weniger Jahre (nur nebenbei bemerkt: unter der Schriftleitung von Peter Sacher und mir 1990-1995) zu einem anerkannten Forum für Arachnofaunistik in Mitteleuropa entwickelte.

In einem Rundbrief der AAB vom 12.11.90 schrieb Theo: "Ich verspreche Euch/Ihnen, dass ich nächstes Jahr nicht mehr so viel Engagement für die Arbeitsgemeinschaft aufbringen werde ...... die Grenze meiner Belastbarkeit ist zur Zeit erreicht bzw. überschritten". Er hat sein "Versprechen" nicht gehalten, er war vom Gründungstag 1990 an bis 1996 Sprecher der SARA, danach Vorsitzender der AraGes bis 2004. Was Theo Blick in diesen
14 Jahren für uns Kollegen und für das Image der Arachnologie geleistet hat, wissen wohl alle, die diesen Artikel lesen. Es muss hier nur grob angerissen werden. Er verhandelte mit Behörden, kümmerte sich um die Durchsetzung vernünftiger Untersuchungsstandards (und vernünftiger Bezahlung) bei Auftragsuntersuchungen, er vernetzte die Arachnologen europaweit untereinander, war unser Informationszentrum, kopierte unermüdlich aus seinem bewunderungswürdigen Literaturfundus, versorgte uns mit dem Zoological Record, mit Arthropoda Selecta, gab uns Literaturtipps, peitschte uns an, Daten zu liefern für die Erstellung von Checklisten, Roten Listen, Verbreitungskarten usw. usw. - und war eigentlich immer da, wenn man Fragen und Probleme hatte.

Wenn die Laudatio für Theo eher wie eine kleine Historie der Entstehung der AraGes ausfällt, dann zeigt das, welche zentrale Rolle als Integrator und Motor er spielte (wobei er noch 1994 zu denen gehörte, die die Gründung einer "Deutschen Arachnologischen Gesellschaft" ablehnten).

Theo Blick ist 2004 aus dem Vorstand der AraGes ausgeschieden und übergewechselt in die Schriftleitung der AraMit. Glaubt er wirklich, damit seine Arbeitsbelastung zu verringern - schafft er es eigentlich wirklich, sein Engagement zu reduzieren? Man wird sehen.

Die AraGes dankt Theo Blick jedenfalls einstweilen für seinen Einsatz für die Sache der Arachnologie in den vergangenen 16 Jahren!

E. Bauchhenß 